\title{
Molecular Association Complexes of Some Ionene Polymers with Thiourea
}

\author{
Tetsuo Tsutsui and Takehide TANAKA \\ Department of Applied Chemistry, Faculty of Engineering, \\ Kyushu University, Fukuoka, Japan.
}

(Received February 7, 1976)

\begin{abstract}
The molecular association complexes of aliphatic $6, n$-ionenes: $\left.+\left(\mathrm{CH}_{2}\right)_{6}-\mathrm{N} \oplus\left(\mathrm{CH}_{3}\right)_{2} \mathrm{Br} \ominus--\left(\mathrm{CH}_{2}\right)_{n}-\mathrm{N} \oplus\left(\mathrm{CH}_{3}\right)_{2} \mathrm{Br} \ominus\right\}_{p}^{-}, n=3,4,5,6$, and 10; with thiourea were obtained. Their elemental analyses were consistent with association of only one molecule of thiourea per quaternary ammonium nitrogen site. The IR spectra of the ionene complexes resembled the spectrum of the noninclusion-type $2: 1$ thiourea complex of hexamethylene-bis(cetyldimethylammonium) dibromide rather than that of the well-defined thiourea inclusion compound of cetyltrimethylammonium bromide. The IR band at $710-730 \mathrm{~cm}^{-1}$ turned out to be useful in distinguishing the ionic noninclusion-type thiourea complexes from the inclusion compounds. The melting temperatures measured by DSC lay between $130^{\circ} \mathrm{C}$ and $150^{\circ} \mathrm{C}$. A typical even-odd alternation was recognized: when $n$ was even, the melting temperature of a complex was higher by $c a .10^{\circ} \mathrm{C}$ than when $n$ was odd.
\end{abstract}

KEY WORDS Ionene Polymers / Molecular Association Complexes / Thiourea / Inclusion Compounds / Ionic Complex / Infrared Spectra / Melting Temperatures /

The crystalline molecular complexes of polymers with urea (U) or thiourea (TU) can be classified into several kinds. Polyethylene and aliphatic polyesters form so-called inclusion compounds (or adduct) with U. ${ }^{1,2}$ Poly(ethylene oxide), on the other hand, forms complexes with $U$ and $T U$, which are not regarded as inclusion compounds. ${ }^{3,4}$ The TU complexes of poly(vinylpyrrolidone) and poly ( $\gamma$-benzyl L-glutamate) are known, but their structures are uncertain. $^{5}$

As for ionic guest molecules, quaternary ammonium salts with a long alkyl chain, such as cetyltrimethylammonium bromide (CTAB), form inclusion compounds with $\mathrm{TU}^{6}$ The TU-complex of pyridinium bromide, $\mathrm{C}_{5} \mathrm{H}_{5} \mathrm{NHBr} \cdot 2 \mathrm{TU}$, however, resembles the TU-complexes of metal halides, MX-4TU, as evidenced by X-ray diffractions. The nature of the hydrogen bonding of TU in these inorganic and pyridinium complexes are considered to be similar. ${ }^{7,8}$ We refer to these complexes as ionic-type complexes, in this paper. Because aliphatic ionenes possess both relatively long methylene chains and many quaternary ammonium cation sites in the skeletons, these polymers might give inclusion compounds and/or ionic-type complexes.

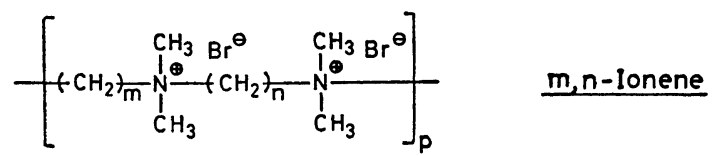

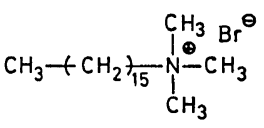

CTAB

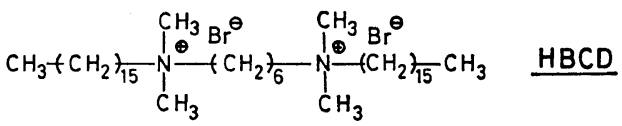

Figure 1. $m, n$-ionenes and their model quaternary ammonium compounds. 


\section{T. Tsutsui and T. TANAKA}

Recently, we found that the $6, n$-ionenes of the general formula given in Figure 1 formed crystalline molecular complexes with TU. In order to elucidate the structures of the ioneneTU complexes, we examined their IR spectra and $\mathrm{X}$-ray diffraction patterns and compared them with those of the TU complexes of CTAB and hexamethylene-bis(cetyldimethylammonium) dibromide (HBCD) - the former is a well-defined inclusion compound, ${ }^{6}$ and the latter is an ionictype complex with the stoichiometry of HBCD. 2TU.

\section{EXPERIMENTAL}

\section{Materials}

By the addition polymerization of $N, N, N^{\prime}$, $N^{\prime}$-tetramethylhexamethylenediamine and $\alpha, \omega$ dibromoalkanes, $6, n$-ionenes $(n=3,4,5,6$, and 10) were prepared. ${ }^{9}$ Intrinsic viscosities of these polymers measured in $0.4-M \mathrm{KBr}$ aqueous solutions at $25.0^{\circ} \mathrm{C}$ were $0.17 \mathrm{~d} l / \mathrm{g}$ (6,3-ionene), $0.20(6,4-), 0.30(6,5-), 0.25(6,6-)$, and 0.28 $(6,10-)$. The molecular weight of the 6,6-ionene was calculated to be $3.1 \times 10^{4}$ from the $[\eta]-\bar{M}_{\eta}$ relationship of 6,6-ionene reported by Casson and Rembaum. ${ }^{10}$

CTAB (commercial reagent) was recrystallized from ethyl acetate. HBCD was obtained by the reaction of $N, N, N^{\prime}, N^{\prime}$-tetramethylhexamethylenediamine with cetyl bromide in ethanol. It was then recrystallized twice from ethanol. TU was recrystallized from methanol.

\section{Formation of Molecular Association Complexes}

Equal volumes of $1-\%$ methanol solutions of each ionene polymer and 5-\% methanol solution of TU were mixed at room temperature. The mixture was then placed in a refrigerator ( $c a$. $5^{\circ} \mathrm{C}$ ) and allowed to stand for a week. The complexes of 6,3-, 6,4-, 6,5-, and 6,6-ionenes were obtained as milky precipitates. No precipitation occurred in the solution containing 6, 10-ionene and TU. In this case, a saturated TU solution was used in place of the 5-\% TU solution, and the complex of 6,10-ionene was obtained. The solution containing the milky precipitate was centrifuged and the solvent was decanted, and the remaining precipitate was washed with a small amount of cold methanol.
The mixture was centrifuged again and the solvent was decanted. Complexes which gave reproducible values of elemental analyses were obtained after three repetitions of the above procedure, except in the case of the 6,10-ionene complex. Preparation of the complexes using several different TU or polymer concentrations was also examined. The compositions of the complexes were not affected by $\mathrm{TU} /$ polymer mole ratio.

The complexes of CTAB and HBCD were made by the following method. In a $2-\% \quad 2-$ propanol solution of TU, $1-\%$ by weight of CTAB or HBCD was dissolved by heating, and the solution was allowed to stand for three days at room temperature. The CTAB complex and the $\mathrm{HBCD}$ complex crystallized as prisms and thin plates, respectively.

\section{Measurements}

IR spectra were recorded by the $\mathrm{KBr}$ disk method on a Perkin-Elmer 337 type spectrophotometer. The spectra by the $\mathrm{KBr}$ disk method were compared with those by the nujol mull method in order to examine the possibility of formation of TU complex with $\mathrm{KBr}{ }^{11}$ No difference was observed between the two spectra.

The wide angle X-ray diffraction patterns of powdery samples were recorded on flat photographic films by using a nickel-filtered $\mathrm{Cu}-\mathrm{K} \alpha$ radiation.

Thermal data (DSC) were obtained using a Rigakudenki differential scanning calorimeter. Powdery samples $(6 \mathrm{mg})$ were used, and the heating rate was $10^{\circ} \mathrm{C} / \mathrm{min}$.

\section{RESULTS AND DISCUSSION}

The results of the elemental analyses of the complexes of $6, n$-ionenes are shown in Table I together with those of the CTAB complex and the HBCD complex. The calculated values for the polymer complexes in Table I correspond to the structures in which one TU molecule associates with each quaternary ammonium ion site of the polymers $(1: 1$ complex). The correspondence between the experimental and the calculated values was fairly good for four polymer complexes. We failed to get reproducible values of the composition of the 6,10-ionene complex owing to the difficulty in removing the 
remaining free TU. This correspondence brought had nearly $100 \%$ crystallinity. We must keep us to the hypothesis that the structures of the in mind, however, that the mere coincidence ionene complexes belonged to the category of of the average compositions of the ionene comionic complexes, and that the polymer complexes plexes with the assumed uniform structures does

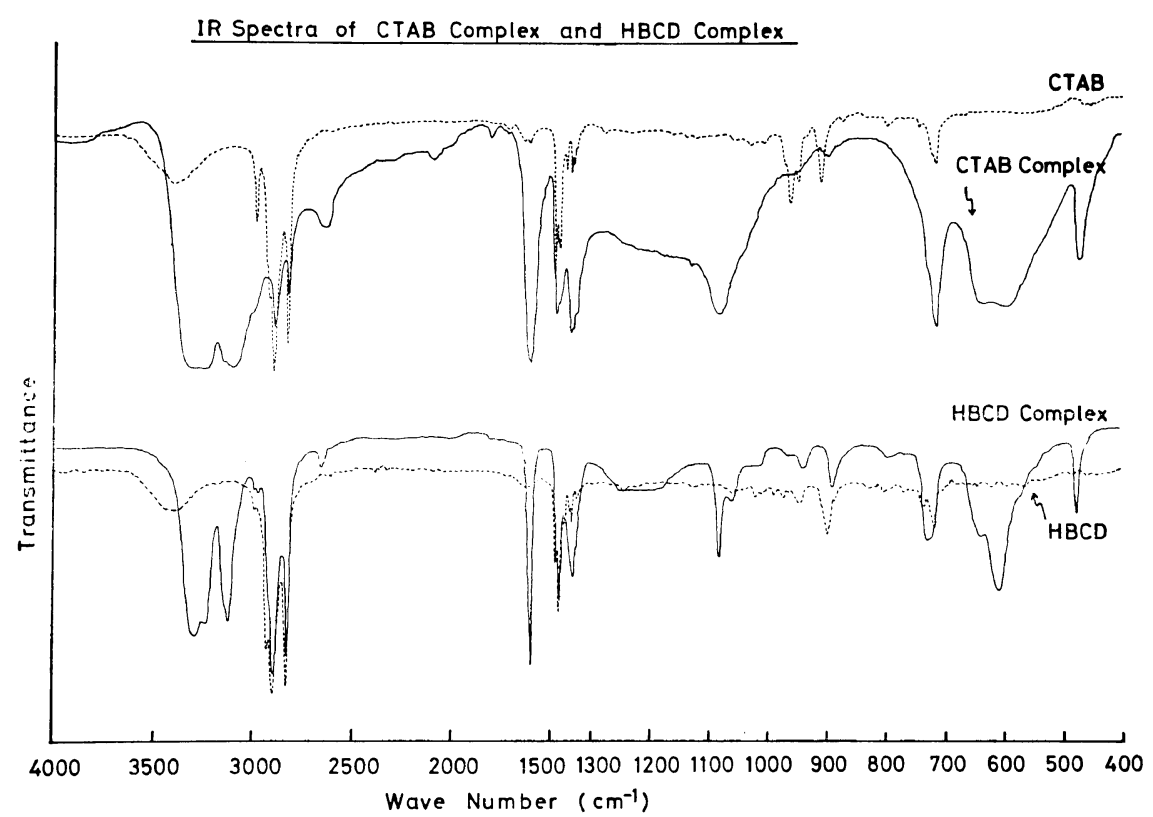

Figure 2. IR spectra of $\mathrm{CTAB}$ complex and $\mathrm{HBCD}$ complex. The broken lines show the spectra of their component materials.

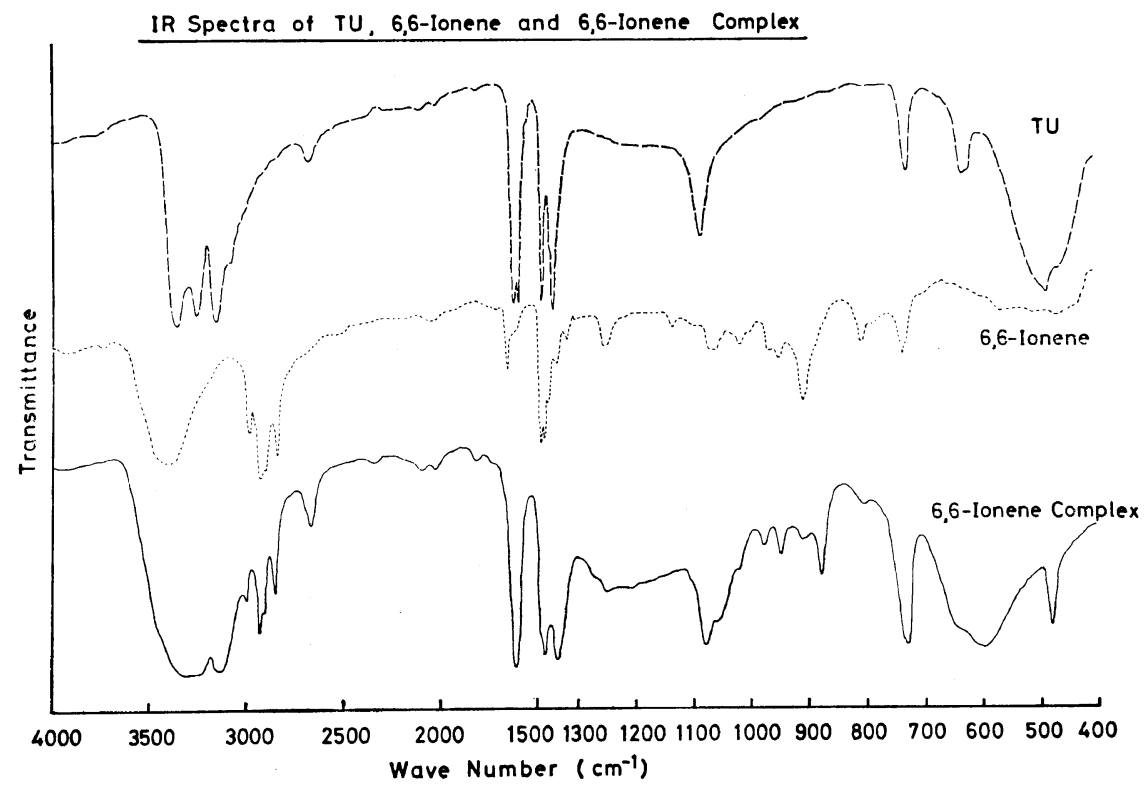

Figure 3. IR spectra of TU, 6,6-ionene, and 6,6-ionene complex. 


\section{T. Tsutsui and T. TANAKA}

not necessarily rule out the possibility that only complex in Table I correspond to the structures part of every ionene chain is included by TU CTAB.10TU and HBCD.2TU, respectively. and the remainder is almost bare. The calculated Schlenck reported the relation between the chain values of the CTAB complex and the HBCD length of a guest molecule and the number of TU

Table I. Elemental analyses of molecular complexes

\begin{tabular}{cccccccc}
\hline \multirow{2}{*}{ Complex } & \multicolumn{3}{c}{ Calculated, $\%$} & & \multicolumn{3}{c}{ Found, \% } \\
\cline { 2 - 6 } \cline { 5 - 7 } & $\mathrm{C}$ & $\mathrm{H}$ & $\mathrm{N}$ & & $\mathrm{C}$ & $\mathrm{H}$ & $\mathrm{N}$ \\
\hline 6,3-ionene & 34.22 & 7.27 & $15.96^{\mathrm{a}}$ & 33.09 & 7.27 & 14.99 \\
6,4-ionene & 35.55 & 7.45 & 15.54 & & 36.10 & 7.56 & 14.96 \\
6,5-ionene & 36.82 & 7.63 & 15.15 & & 36.20 & 7.60 & 14.92 \\
6,6-ionene & 38.02 & 7.80 & 14.78 & & 36.33 & 7.63 & 14.87 \\
6,10-ionene & 42.29 & 8.39 & 13.45 & & - & - & - \\
CTAB & 30.95 & 7.34 & $26.14^{\mathrm{b}}$ & & 30.71 & 7.29 & 26.11 \\
HBCD & 56.50 & 10.56 & $8.98^{\mathrm{c}}$ & & 56.30 & 10.56 & 8.94 \\
\hline
\end{tabular}

a Calculated for $\left(\mathrm{CH}_{2}\right)_{6}\left(\mathrm{CH}_{2}\right)_{n}\left(\mathrm{CH}_{3}\right)_{4} \mathrm{~N}_{2} \mathrm{Br}_{2} \cdot\left(\mathrm{CSN}_{2} \mathrm{H}_{4}\right)_{2}(n=3,4,5,6$, and 10).

b Calculated for $\mathrm{C}_{16} \mathrm{H}_{33} \mathrm{~N}\left(\mathrm{CH}_{3}\right)_{3} \mathrm{Br} \cdot\left(\mathrm{CSN}_{2} \mathrm{H}_{4}\right)_{10}$.

c Calculated for $\left(\mathrm{C}_{16} \mathrm{H}_{33}\right)_{2}\left(\mathrm{CH}_{3}\right)_{4}\left(\mathrm{CH}_{2}\right)_{6} \mathrm{~N}_{2} \mathrm{Br}_{2} \cdot\left(\mathrm{CSN}_{2} \mathrm{H}_{4}\right)_{2}$.

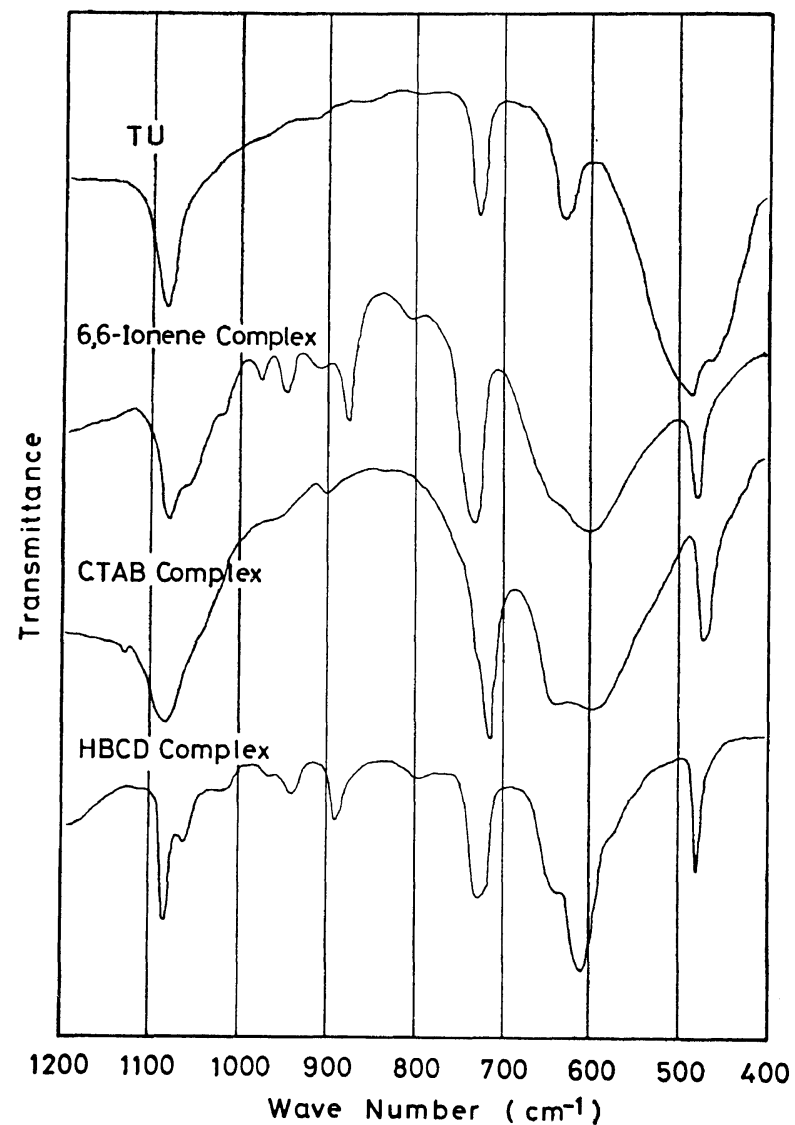

Figure 4. Comparisons of IR spectra of TU, 6,6-ionene complex, CTAB complex, and HBCD complex in the low-wave-number region. 
molecules per guest molecule in the inclusion compounds of $\mathrm{TU} .^{12}$ An application of this relation immediately reveals that the $C T A B$ complex is an inclusion compound, but the HBCD complex is not.

The IR spectra of the CTAB complex and the HBCD complex are shown in Figure 2, along with those of component materials. The spectrum of the CTAB complex coincided with those of inclusion compounds of TU reported previously. ${ }^{13,14}$ Figure 3 shows the spectra of the 6,6-ionene complex, 6,6-ionene, and TU. At a first glance, the spectra of both HBCD complex and 6,6. ionene complex look very similar to those of inclusion compounds. Examined in detail, however, there are distinct differences in the relative intensities of the bands at $2800-3000 \mathrm{~cm}^{-1}$ and in the location of the band at $710-730 \mathrm{~cm}^{-1}$. The difference in the intensities of the bands at $2800-3000 \mathrm{~cm}^{-1}$ is caused by the difference in the mole ratio of $\mathrm{TU}$ and the guest molecules. The band at $710-730 \mathrm{~cm}^{-1}$, where the contribution of a $\mathrm{C}=\mathrm{S}$ stretching vibration is known to be about $50 \%,{ }^{15}$ sharply reflects the type of complexes. The difference in the state of $\mathbf{S}-\mathbf{H}$ hydrogen bonds influences this band. The TU molecules in ionic type complexes are aligned as sheets by hydrogen bonds between a sulfur atom and two hydrogen atoms. On the other hand, the sulfur atom in the inclusion compounds enter into hydrogen bonds with four equivalent hydrogen atoms, thus forming a three

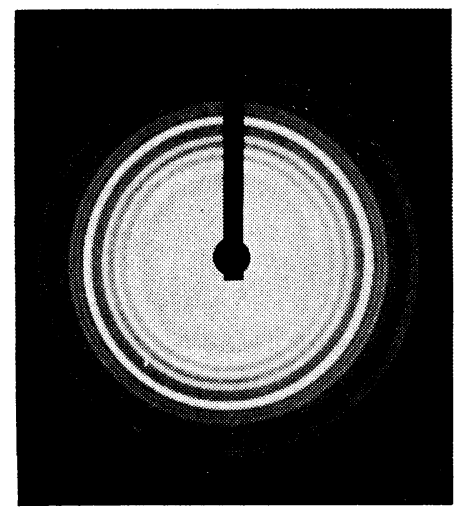

(a)

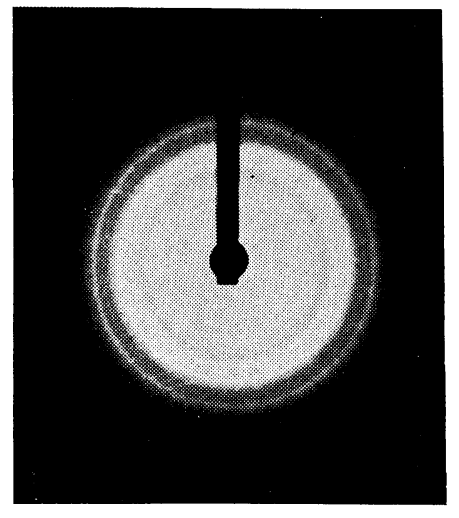

(b)

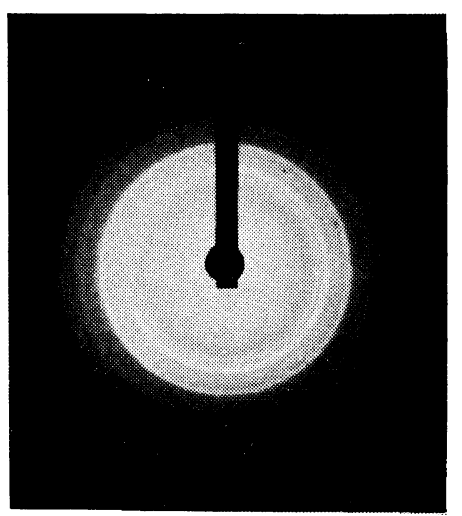

(c)

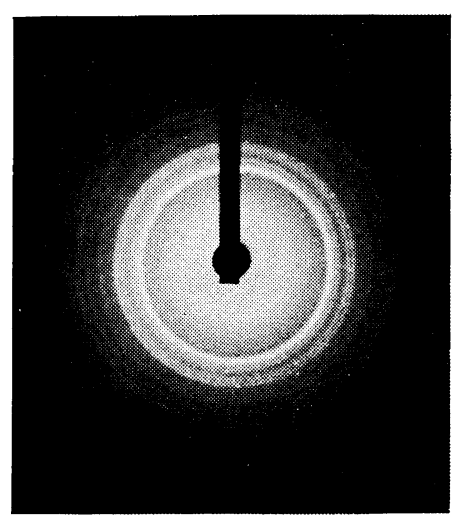

(d)

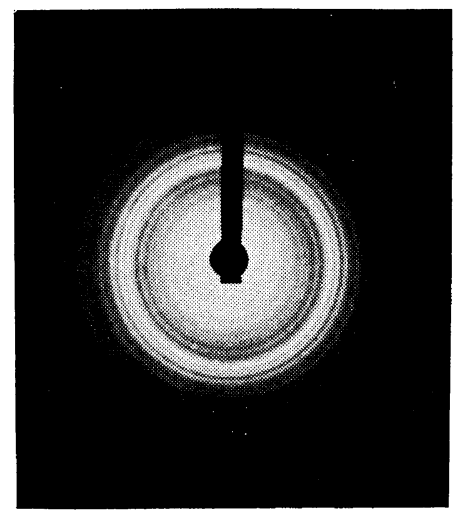

(e)

Figure 5. X-ray diffraction patterns of complexes and their component materials: (a) TU: (b) 6,6ionene; (c) 6,6-ionene complex; (d) CTAB complex; (e) HBCD complex. 


\section{T. Tsutsui and T. TANAKA}

dimensional network of TU molecules. ${ }^{16,8}$ Whereas the band of inclusion compounds appear at $715 \mathrm{~cm}^{-1}, 13,14$ the band of the HBCD complex is found at $730 \mathrm{~cm}^{-1}$. Since the band of the 6,6-ionene complex lies also at $730 \mathrm{~cm}^{-1}$, the polymer complexes are considered to have a structure similar to that of the HBCD complex, and the formation of an uniform 1:1 complex is further supported (Figure 4).

Figure 5, shows the X-ray diffraction patterns of TU, 6,6-ionene, the 6,6-ionene complex, the CTAB complex, and the HBCD complex. The diffraction pattern of the 6,6-ionene complex does not contain patterns due to 6, 6-ionene or TU itself, and this clearly indicates the formation of a new crystalline association complex. Inclusion compounds of TU are known to crystallize in hexagonal lattice with $a=15.8 \mathrm{~A}$ and $c=$ $12.5 \mathrm{~A}^{16}$ It was impossible to ascertain whether the diffraction pattern of the CTAB complex could be indexed by the hexagonal lattice, owing to the limited number of reflections. Comparison of the diffraction patterns of the three complexes brings us to the conclusion that the crystal structures of the HBCD complex and the 6,6ionene complex differ from that of the CTAB complex i.e., differ from that of an inclusion compound.

The DSC thermograms of the polymer complexes are shown in Figure 6. Shoulders were observed in the melting peaks of the 6,3-ionene complex and the 6,4-ionene complex. Rapid cooling in the process of crystallization in methanol might have given some imperfections in the crystals of the complexes. The small peak just below the main melting peak of the 6,10-ionene complex is considered to originate from partial solution and recrystallization during the process of washing the precipitated complex with fresh methanol. Though the sample of the 6,10-ionene complex contained as impurities small amounts of free $\mathrm{TU}$, the main melting peak of the complex did not depend on the amounts of the free TU. The peak temperature of each thermogram was plotted against $n$, the number of carbon atoms in the skeletal chains of $6, n$-ionene, in Figure 7. The melting temperatures of the complexes with even $n$ are approximately ten degrees higher than those of the complexes with odd $n$. This is the particular case of $m=6$ in $m, n$-ionene complexes, but we expect that the alternation of melting temperatures of this sort might be present in the series with another $m$ values. Similar odd-even re-

DSC Thermograms of $6, n$-Ionene Complexes
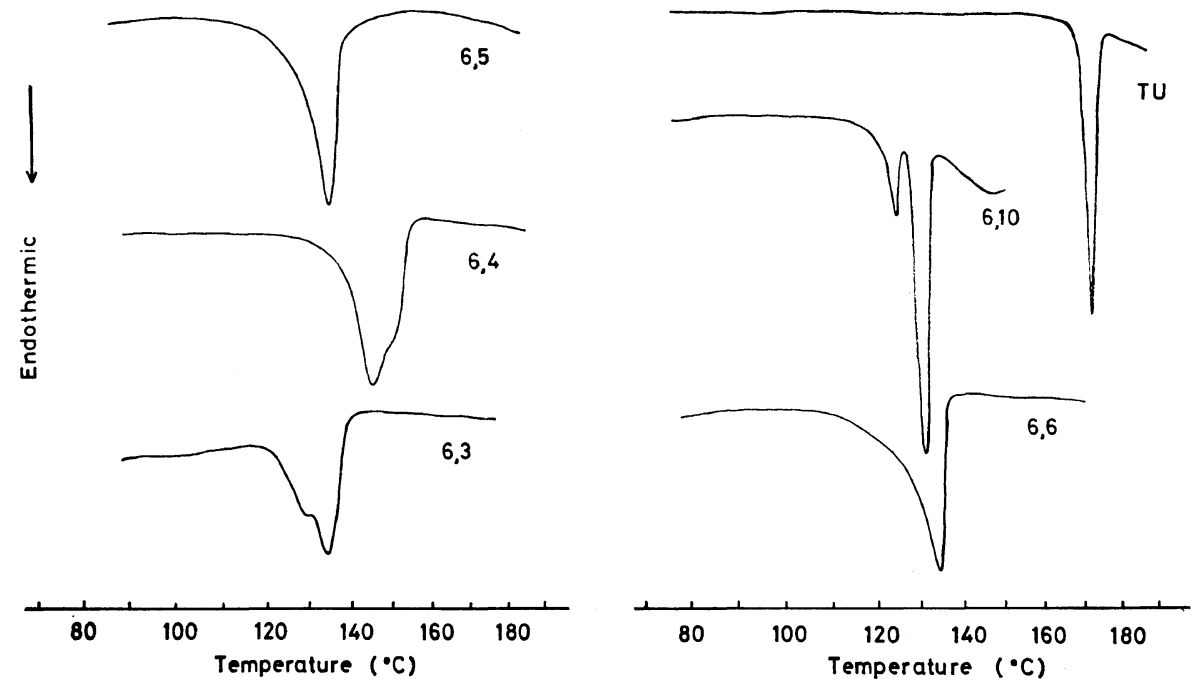

Figure 6. DSC thermograms of $6, n$-ionene complexes and TU. $m, n$ in the figure indicates the $m, n$-ionene complex. 


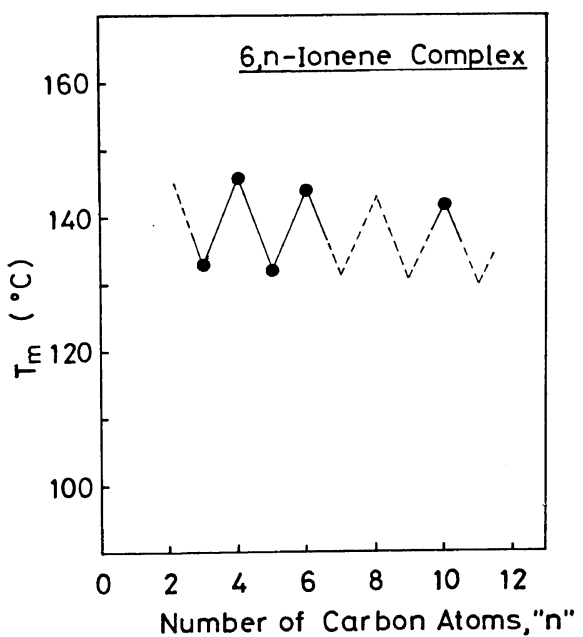

Figure 7. Melting temperatures of $6, n$-ionene complexes.

lations in melting temperatures are well-known phenomena for aliphatic polyesters, polyamides, and polyurethanes. ${ }^{17,18,19}$ Differences in the ease of hydrogen bonding are believed to be responsible for this odd-even alternation. A similar interpretation may also hold in the case of the $6, n$-ionene complexes. The stability of hydrogen bonds of TU molecules as well as the ease of packing of the ionic parts and the methylene sequences of the polymers, influence the melting temperatures of the polymer complexes.

The presence of the odd-even relation in the melting of the polymer complexes gives further evidence that the structure of the polymer complexes differs from inclusion compounds. Since there is little interaction between the hexagonal lattice of TU and the guest molecules in inclusion compounds, a small change in the shape of the guest molecules should not greatly influence the melting of the hexagonal lattice of TU, and the odd-even relation in melting temperatures is not expected.
Aknowledgment. The authors wish to express their gratitude to Dr. R. Tanaka of Kyushu University for helpful discussions. This work was partly supported by the Grant-in-Aid for Scientific Research from the Ministry of Education, Japan, and also by a grant from Association for Promotion of Industrial Technology, Asahi Glass Co. Ltd.

\section{REFERENCES}

1. K. Monobe and F. Yokoyama, J. Macromol. Sci.-Phys., 38, 277 (1973).

2. R. Mayer, Bull. Soc. Chim. France, 2Sss (1966).

3. F. E. Bailey and H. G. France, J. Polym. Sci., 49, 397 (1961).

4. H. Tadokoro, et al., ibid., Part-B, 2, 363 (1964).

5. G. Hild, Rev. Gen. Caut. Plast., 46, 771 (1969).

6. D. E. Cadwallader and J. B. Richards, J. Amer. Oil Chemists' Soc., 42, 337 (1965).

7. J.C. A. Boeyens and F. H. Herbstein, Inorg. Chem., 6, 1408 (1967).

8. M. R. Truter and B. L. Vickery, Acta Crystallogr. B28, 387 (1972).

9. N. Noguchi and A. Rembaum, J. Polym. Sci., Part-B, 7, 383 (1969).

10. D. Casson and A. Rembaum, Macromolecules, 5, 75 (1972).

11. J. E. Stewrt, J. Chem. Phys., 26, 248 (1957).

12. W. Schlenck, Ann., 573, 142 (1951).

13. R. Mecke and W. Kutzelnigg, Zeit. Anal. Chem., 170, 114 (1959).

14. M. Nishikawa, H. Mima, and T. Kanzawa, Chem. Pharm. Bull. (Tokyo), 10, 1199 (1962).

15. G. B. Aitkin, J. L. Duncan, and G. P. McQuillan, J. Chem. Soc., Sect. A, 2695 (1971).

16. H. U. Lenné, Acta Crystallogr. 7, 1 (1954).

17. C. S. Fuller and C. L. Erickson, J. Amer. Chem. Soc., 59, 344 (1937).

18. D. D. Coffman, G. J. Berchet, W. R. Peterson, and E. W. Spanagel, J. Polym. Sci., 2, 306 (1947).

19. O. Bayer, Angew. Chem., 59, 257 (1947). 